\title{
苂光猝灭响应的溶解氧有机改性溶胶-凝胶 传感膜的构筑
}

\begin{abstract}
辛玲玲 ${ }^{(1)}$ 肖来龙 ${ }^{(1)}$ 赵 丽 $^{(1)}$ 陈 曦 ${ }^{(12) *}$ 王小如 $^{(1)}$
(1) 厦门大学化学化工学院化学系, 现代分析科学教育部重点实验室, 厦门 361005; (2) 厦门大学近海海洋环境科学国家重点实验室,

厦门 361005.* 联系人, E-mail: xichen@xmu.edu.cn )

摘要 利用二甲基二甲氧基硅烷为有机改性剂, 制备溶胶-凝胶, 并通过对氧苂光猝灭指示剂 4,7-二苯 基-1,10-令菲咯啉钉 $\left(\mathrm{Ru}(\mathrm{dpp})_{3}\left(\mathrm{ClO}_{4}\right)_{2}\right)$ 的包埋, 构筑以 $\mathrm{Ru}(\mathrm{dpp})_{3}\left(\mathrm{ClO}_{4}\right)_{2}$ 为苂光指示剂的氧传感敏感膜. 利 用蓝光发光二极管 (LED, $\lambda_{\max }=475 \mathrm{~nm}$ ) 为激发光源, 考察了氧敏感膜的苂光行为, 并以此进行了水体 中溶解氧含量的检测. 传感膜对水溶液溶解氧的最低检出限为 $0.2 \mu \mathrm{g} / \mathrm{mL}$, 测定的相对标准偏差为 $2 \%$, 线性范围为 $0.5 \sim 16 \mu \mathrm{g} / \mathrm{mL}$ ，响应时间 $\left(t_{95}\right)$ 为 $60 \mathrm{~s}$, 敏感膜使用寿命大于 10 个月.
\end{abstract}

关键词 有机改性溶胶-凝胶 苂光猝灭 氧传感 钉配合物

水体中溶解氧含量的测定在工业、医疗卫生、生 物、环境和水产养殖等诸多方面有着重要的意义, 在 环境检测中, 其含量是代表水体洁净程度的重要指 标之一. 研制具有可靠、可逆和便宜的水体溶解

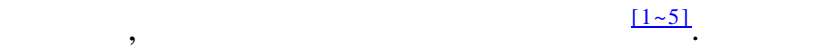
配位化合物, 尤其是 4,7-二苯基-1,10-邻菲咯啉钉 $\left(\mathrm{Ru}(\mathrm{dpp})_{3}\left(\mathrm{ClO}_{4}\right)_{2}\right)$ 具有光和热稳定性良好、合成方便 和合成成本较低等优点, 较多地应用在氧的光化学 传感器中 [6 9]. 由于溶胶-凝胶(sol-gel)具有良好的光 学通透性、机械特性和化学稳定性, 同时由于制备过 程中温和的反应条件，灵活而方便的制备方式，以及 对光敏物质达到分子水平上的均匀包埋而备受人们 的关注. 近年来, 利用溶胶一凝胶材料进行钉配合物 的包埋, 并实现水体中溶解氧的光化学测定已有报 道 [10 15]. 但直接利用无机硅氧烷水解进行sol-gel制 备, 存在着基质材料机械柔韧性较差, sol-gel基质的 极性与苂光试剂的极性不相匹配, 导致试剂渗漏等 问题. 本文报道利用有机硅氧烷二甲基二甲氧基进 行sol-gel的有机改性制备, 以 $\mathrm{Ru}(\mathrm{dpp})_{3}\left(\mathrm{ClO}_{4}\right)_{2}$ 为氧猝 灭苂光指示探针, 制备了氧敏感膜; 并利用蓝光发光 二极管(LED)为激发光源, 考察了 $\mathrm{Ru}(\mathrm{dpp})_{3}\left(\mathrm{ClO}_{4}\right)_{2}$ 包 埋在有机改性 sol-gel膜内对水体中溶胶氧的响应及 苂光光谱行为的变化情况; 利用构建的流动测定系 统进行了水体中溶解氧的测定.

\section{1 实验}

( i ) 化学试剂和主要仪器. 实验所用四甲氧基 硅烷(TMOS)购自中国医药(集团)上海化学试剂公司;
甲基三甲氧基硅烷(MTMS)、苯基三甲氧基硅烷 (PTMS)和二甲基二甲氧基硅烷(DiMe-DMOS)购自瑞 士 Fluka 公司; 4,7-二苯基 -1,10-邻菲咯啉钉 $\left(\mathrm{Ru}(\mathrm{dpp})_{3}\left(\mathrm{ClO}_{4}\right)_{2}\right)$ 由本实验室合成，其结构式见图 1. 实验中的苂光光谱通过 F-4500 (Hitachi, Japan)苂光 光度计扫描获得.

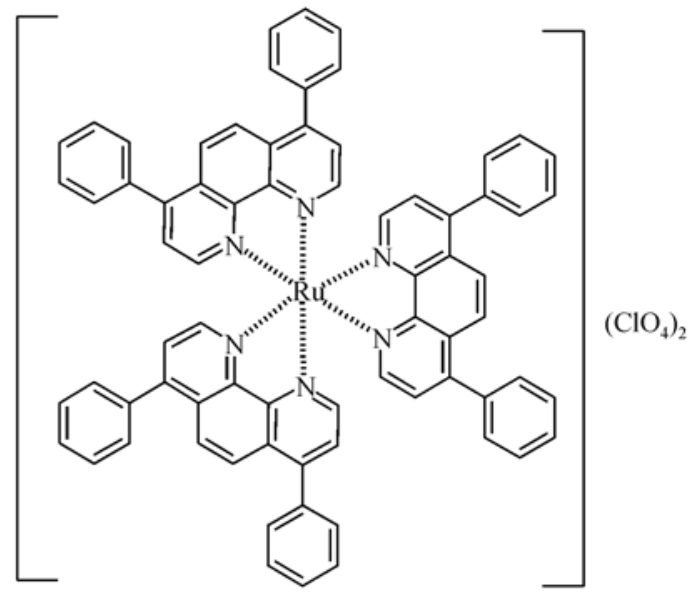

图 $1 \mathrm{Ru}(\mathrm{dpp})_{3}\left(\mathrm{ClO}_{4}\right)_{2}$ 的化学结构

两套 GF-4300 流量控制计(Gilmont Instruments Inc) 经 J\&W ADM1000 智能流量计校正后, 分别用 于准确控制氧气和氮气的流量大小. 不同浓度的气 态氧由准确控制氧气、氮气的流速按一定比例混合后 通入测量池, 而不同浓度的溶解氧标液由不同氧气 含量的氮气、氧气混合气体饱和蒸馏水溶液配制. 实 验主要采用测定氧传感膜在氮气饱和蒸馏水中的苂 光强度 $I_{\mathrm{N}_{2}}$ 与在氧气饱和蒸馏水中的苂光强度 $I_{\mathrm{O}_{2}}$ 的比 
值 $R$, 即 $R=I_{\mathrm{N}_{2}} / I_{\mathrm{O}_{2}}$, 来表征氧传感膜对氧响应灵敏度 的大小, 从而进行各种实验条件的优化与选择.

(ii ) 敏感膜制备. 实验所用的铺膜载基玻片 (15 mm× $30 \mathrm{~mm} \times 0.15 \mathrm{~mm}$ )使用前用浓硝酸浸泡 24 $\mathrm{h}$ 后分别用蒸馏水和乙醇冲洗, 于 $80^{\circ} \mathrm{C}$ 烘干 $2 \mathrm{~h}$ 后 备用.

按一定体积比量取 TMOS 和 DiMe-DMOS 置于 开口瓶中, 加入适量浓度的 $\left[\mathrm{Ru}(\mathrm{dpp})_{3}\right]^{2+}$ 的四氢呋 喃(THF)溶液, 旋涡振荡 $1 \mathrm{~min}$ 后, 再逐滴振荡加入 $0.01 \mathrm{~mol} / \mathrm{L} \mathrm{HCl}$, 调节体系的 $\mathrm{pH}$, 将瓶子于 $60^{\circ} \mathrm{C}$ 下敞 口磁摚拌水浴加热 $3 \mathrm{~h}$. 取以上所制得的凝胶液 60 $\mu \mathrm{L}$, 均匀涂布于事先经乙醇和硝酸处理的玻片上成 膜, 于烘箱中干燥 $12 \mathrm{~h}$ 后备用. 敏感膜厚度利用场发 射扫描电子显微镜(FE-SEM; JSM-7400F, JEOL, Japan)检测, 膜平均厚度为 $(35 \pm 3) \mu \mathrm{m}(n=10)$.

(iii) 氧苂光猝灭测定装置. 为提高敏感膜的苂 光强度的变化值, 以及对氧检测的灵敏度, 同时为进 一步简化敏感流通池的结构, 在传感探头设计中使 用互成 $90^{\circ}$ 入射的 4 个蓝色 LED (图 2(b)). 感应光纤 头正对着氧传感膜, 膜中的钉配合物被 LED 直接照 射激发产生苂光. 苂光信号通过光纤传导经通带滤 光片滤净激发光后再进入光电倍增管. 由光电倍增 管输出的电流信号先转化为电压信号, 再由 A/D 转 换器变换为数字信号, 最后由计算机记录、分析和处 理. 实验所选用的通带滤光片的透光波带宽为 585 605 nm, 最大通过波长 $595 \mathrm{~nm}$. 该滤光片能有 效消除激发光的干扰，保证对发射光的最小吸收(图 2(a)). 水样品进样口使用 $100 \mu \mathrm{m}$ 不锈钢砂芯, 样品 在进入检测池前, 经过内装有孔径 $20 \mu \mathrm{m}$ 尼龙膜的过
滤圆盘, 以进一步减少水体杂质在膜片和光学窗口 的吸附. 实验结束后, 流路使用蒸馏水冲洗 $15 \mathrm{~min}$.

\section{2 结果与讨论}

\section{1 溶胶-凝胶前驱体成分的优化}

前驱体的选择对钉配合物的苂光猝灭有很大的 影响. 通常在有机改性 sol-gel 制备过程中, 使用带有 烷基取代的有机硅氧烷(MTMS, PTMS 或 DiMeDMOS)单体或聚合物与硅氧烷, 如四甲氧基硅烷 (TMOS)或四乙氧基硅烷(TEOS)等水解聚合. 实验结 果表明, 在加入相同摩尔比的情况下, 对比使用带有 一个甲基或苯基取代的 MTMS 或 PTMS, DiMeDMOS 所获得的传感膜基质材料具有柔韧性好、响应 速度快和氮氧饱和苂光猝灭比高等优点(表 1). 由于 氧分子中双原子的对称性, 氧分子的极性极小, 这使 得氧分子易于在非极性环境或膜中渗透. DiMeDMOS 含有两个取代的甲基, 与 TMOS 水解形成的 sol-gel 膜, 具有比 MTMS 或 PTMS 更高的疏水性, 而 使所获得的基质材料对氧具有更好的亲和性、对氧易 于渗透的特性, 使得氧容易与固定于 DiMe-DMOS 膜 中的 $\mathrm{Ru}(\mathrm{dpp})_{3}\left(\mathrm{ClO}_{4}\right)_{2}$ 指示剂产生苂光猝灭作用, 而获 得对氧有较高灵敏度和较短响应时间的敏感膜. 同 时由于 DiMe-DMOS 存在两个难键合和水解的甲基, 膜基质中的 Si-O-Si 结构多呈线状或平面, 减少了膜 的内张力, 使得膜的柔韧性增加.

敏感膜的极性对氧的响应时间和检测灵敏度有 很大的影响, 实验发现通过改变前驱体中 DiMeDMOS 对 TMOS 的相对含量, 可以调整氧传感膜的 极性. 由于 DiMe-DMOS 含量的增加, -Si-O-结构末端

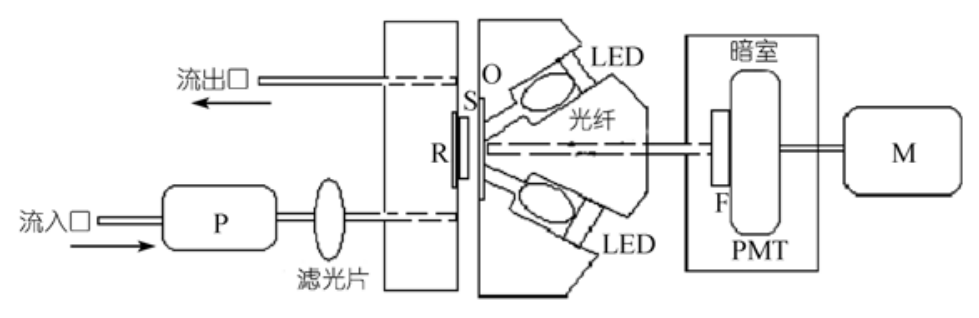

(a)

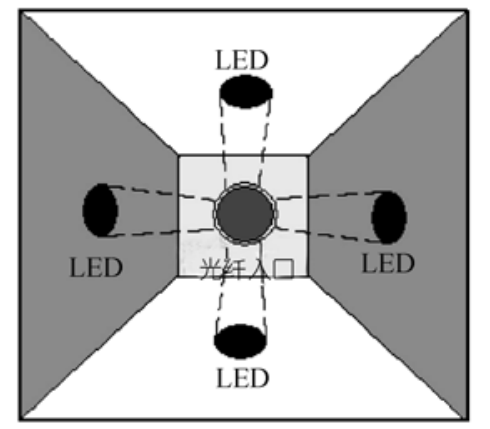

(b)

图 2 氧苂光猝灭测定装置示意图

(a) 氧测定装置设计图; (b) 传感室平面图. P, 蠕动泉; R, 反射镜; S, 氧传感膜; $O$, 光学窗口; F, 干涉滤光片; https LED, 发光三极管; PMT, 光电倍增管; M, 光度计6-921 
表 1 不同前驱体配比时氧传感膜的性质

\begin{tabular}{|c|c|c|c|c|c|c|c|c|}
\hline 编号 & $V(\mathrm{TMOS}) / \mathrm{mL}$ & $V($ DiMe-DMOS $) / \mathrm{mL}$ & $V(\mathrm{MTMS}) / \mathrm{mL}$ & $V(\mathrm{PTMS}) / \mathrm{mL}$ & 机械性能 & 响应时间 $/ \mathrm{s}^{\mathrm{a})}$ & $\begin{array}{c}\mathrm{Ru}(\mathrm{dpp})_{3}\left(\mathrm{ClO}_{4}\right)_{2} \\
\text { 泄漏情况 }\end{array}$ & $R\left(I_{\mathrm{N}_{2}} / I_{\mathrm{O}_{2}}\right)^{\mathrm{b})}$ \\
\hline 1 & 0.3 & 0.4 & 0 & 0 & 柔韧 & 60 & - & 4.8 \\
\hline 2 & 0.3 & 0 & 0.4 & 0 & 坚硬 & 86 & - & 1.3 \\
\hline 3 & 0.3 & 0 & 0 & 0.4 & 坚硬 & 110 & - & 1.5 \\
\hline
\end{tabular}

a) 从氮饱和溶液到氧饱和溶液的响应时间 $\left(t_{95}\right)$; b) 氮饱和与氧饱和溶液的苂光猝灭比

表 2 不同 DiMe-DMOS 含量条件下氧传感膜的响应及特性

\begin{tabular}{|c|c|c|c|c|c|c|c|}
\hline 编号 & $V(\mathrm{TMOS}) / \mathrm{mL}$ & $V($ DiMe-DMOS $) / \mathrm{mL}$ & 机械性能 & 相应时间/s & $\begin{array}{c}\mathrm{Ru}(\mathrm{dpp})_{3}\left(\mathrm{ClO}_{4}\right)_{2} \\
\text { 泄漏情况 }^{\text {a) }}\end{array}$ & $R\left(I_{\mathrm{N}_{2}} / I_{\mathrm{O}_{2}}\right)$ & 检测限/ $/ \mu \mathrm{g} \cdot \mathrm{mL}^{-1}$ \\
\hline 1 & 0.2 & 0.15 & 坚硬 & 60 & $15 \%$ & 4.3 & 0.5 \\
\hline 2 & 0.2 & 0.20 & 微硬 & 54 & $8 \%$ & 4.6 & 0.7 \\
\hline 3 & 0.2 & 0.24 & 柔韧 & 40 & - & 5.2 & 0.3 \\
\hline 4 & 0.2 & 0.28 & 柔韧 & 34 & - & 6.1 & 0.3 \\
\hline 5 & 0.2 & 0.32 & 柔韧 & 30 & - & 7.4 & 0.2 \\
\hline 6 & 0.2 & 0.36 & 黏稠 & 46 & $5 \%$ & 6.9 & 0.3 \\
\hline
\end{tabular}

a) 传感膜在氧饱和溶液中浸泡 $60 \mathrm{~d}$

的羟基被甲基取代的数目增加, 其末端羟基数目减 少, 溶胶一凝胶膜的极性随之减小，从而为溶解氧的 灵敏响应提供了一个更适合的环境. 从表 2 可以看出, 随着 DiMe-DMOS 在前驱体中含量的增大, 传感膜对 氧的响应灵敏度明显增加，同时响应时间减少，膜的 柔韧性增加. 而当 DiMe-DMOS 与 TMOS 的体积比达 到 1.8:1 以上时, 难以成膜, 同时不易完全干燥, 响 应时间有所增长, 响应灵敏度反而有所下降. 研究结 果表明 $62 \%$ 的 DiMe-DMOS 为较合适的加入量.

\section{2 利用 LED 激发的氧敏感膜荧光响应特性}

研究考察了利用最大激发波长为 $475 \mathrm{~nm}$ 的蓝光 LED 为激发光源时, 氧敏感膜的苂光响应特性. 结果 表明, LED 的启动电压为 $2.5 \mathrm{~V}$ 左右, 其发光强度与 半峰宽随施加电压的增大而增加; 当施加 LED 的电 压为 $4.0 \mathrm{~V}$ 时, LED 给出的光强最大, 这时 LED 发光 的半峰宽为 $41 \mathrm{~nm}$ (图 3). 随着施加电压的进一步加 大, LED 的发光强度反向减少, 但发光的半峰宽没有 发生明显的变化. 利用 LED 的激发光, 考察了氧敏 感膜的苂光发射波长, 发现其最大发射波长在 598 $\mathrm{nm}$ 左右, 其 Stock 位移为 $135 \mathrm{~nm}$ (图 4). 虽然实验已 尽量选择半峰宽较小的 LED, 但从图 4 的 LED 激发 与发射光谱中可以看出，激发与发射光之间仍然存 在重叠. 为减少激发光对实际测定的影响, 实验选用 了通透波长为 $595 \mathrm{~nm}$, 通透峰宽为 $20 \mathrm{~nm}$ 的通带滤 光片. 由图 4 可以发现, 虽然通带滤光片的使用降低 了发光波长的强度, 但可以完全消除激发光的影响.

\section{3 氧传感膜的响应}

由于在有机改性 sol-gel 制备过程中加入 DiMe-

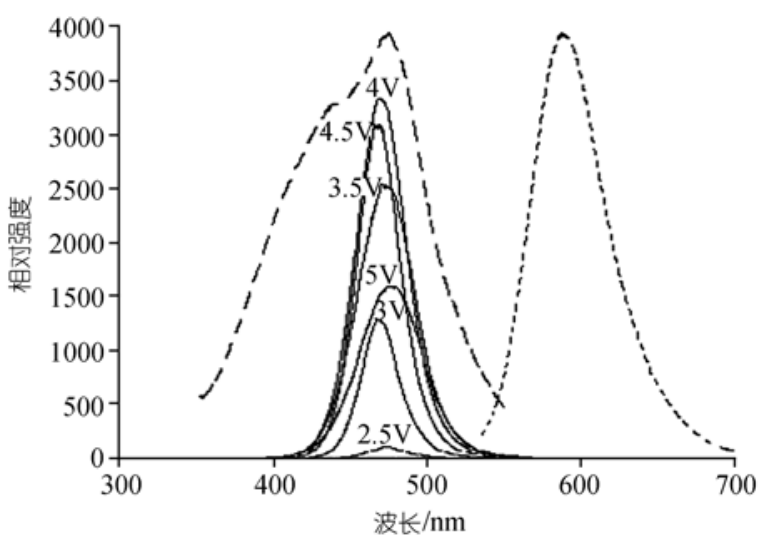

图 3 不同电压下发光二极管的光谱特性以及 $\mathrm{Ru}(\mathrm{dpp})_{3}\left(\mathrm{ClO}_{4}\right)_{2}$ 的吸收(实线)和发射(虚线)光谱的比较

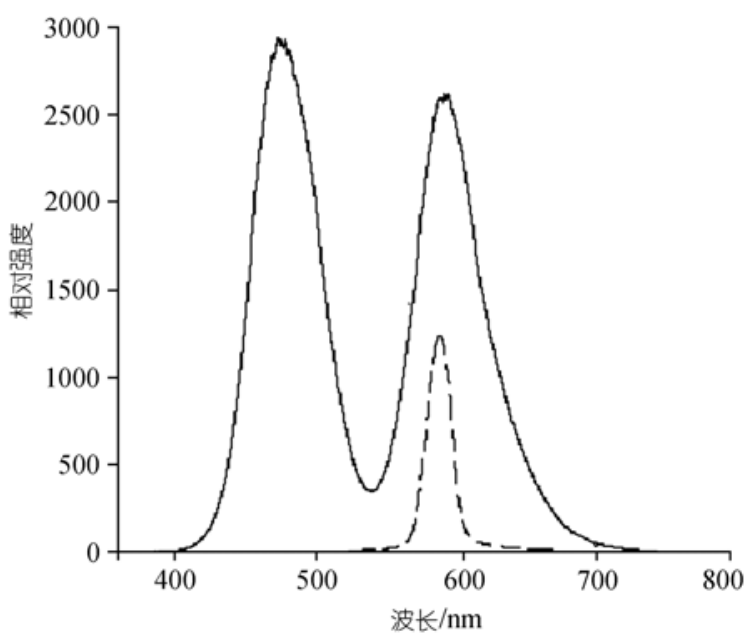

图 4 使用(虚线)与不使用(实线)干涉滤光片情况下 氧传感器苂光信号的比较 
DMOS, 减少了传感膜基质的亲水性, 使水体中的氧 容易以气态形式逸出并进入传感膜, 从而提高敏感 膜对氧的灵敏度, 减少其响应时间 16,17$]$. 图 5 显示测 定初始时的苂光强度为 960 左右, 其强度大小介于氧 和氮饱和溶液的苂光强度之间(苂光强度分别约为 290 和 2060). 由于水溶液中溶解的氧处于与外界空 气氧的交换的平衡状态, 水体中存在一定浓度的氧, 该氧浓度正介于氧和氮饱和溶液的浓度之间。实验 结果表明, 敏感膜的苂光信号强度在氮饱和与氧饱 和溶液更换时, 具有良好的可逆性. 在从氮饱和溶液 更换到氧饱和溶液的过程中, 敏感膜的响应时间约 为 $60 \mathrm{~s}$; 而从氧饱和到氮饱和的响应时间比前一过程 为长, 约为 $200 \mathrm{~s}$. 该现象进一步说明有机改性敏感 膜对氧的亲和程度大于对氮的亲和. 在溶液氮饱和 过程中, 气体氮需要克服敏感膜对氧的吸附并进行 驱除, 该过程需要较长的时间. 另一方面敏感膜的响 应时间还与敏感膜的厚度有关, 较厚的敏感膜对氧 的传质阻力较大, 响应时间增加. 但由于膜的机械性 能的限制, 且考虑氧敏感膜的使用寿命, 实验使用的 敏感膜厚度为 $35 \mu \mathrm{m}$.

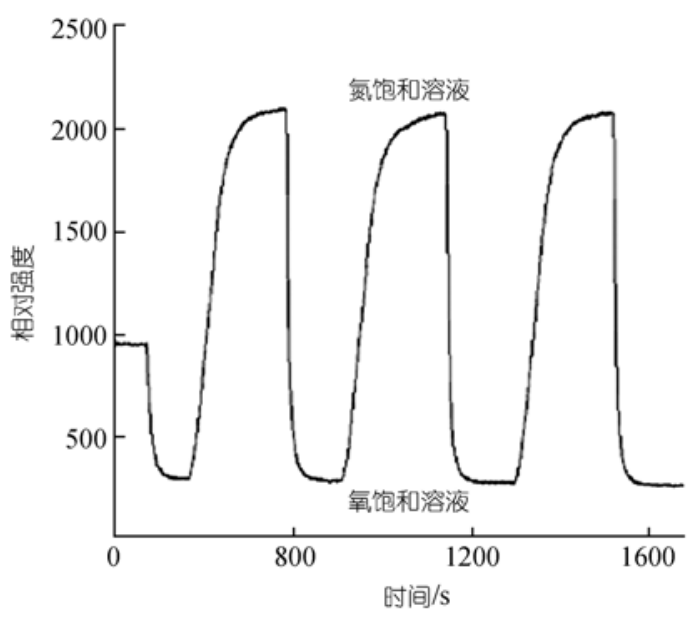

图 5 氧传感膜在氧饱和与氮饱和溶液中的响应曲线

光化学稳定性是氧苂光猝灭敏感膜的一个重要 指标. 实验使用 LED 蓝光光源连续照射苂光指示剂 $\mathrm{Ru}(\mathrm{dpp})_{3}\left(\mathrm{ClO}_{4}\right)_{2}$ 和敏感膜各 $240 \mathrm{~h}$, 然后利用核磁共 振氢谱对比照射前后的 NMR 谱图的变化, 并检测敏 感膜对氧饱和溶液的响应情况. 发现照射前后的 NMR 谱图未见有任何变化, 而敏感膜对 $5.0 \mu \mathrm{g} / \mathrm{mL}$ 氧 的苂光响应值下降 $3 \%$ 左右. 实验结果说明, 菼光试 剂对所选用的激发波长在照射时间内不发生分解或
其他光化学反应, 而对氧灵敏度的变化也在测定误 差之内. 进一步研究发现, 敏感膜浸泡在水中 $60 \mathrm{~d}$, 没有发现指示剂的渗漏. 敏感膜在干燥避光环境中 保存 10 个月, 其对 $5.0 \mu \mathrm{g} / \mathrm{mL}$ 氧的苂光响应值变动范 围为 $\pm 5 \%$. 由于在敏感膜制备中加入 DiMe-DMOS, 增加了膜的疏水性, 敏感膜不对氢离子浓度响应, 因 此测定体系的 $\mathrm{pH}$ 变化, 不影响敏感膜对氧的响应.

\section{4 水体中溶解氧的测定}

苂光猝灭的氧光化学传感检测原理主要基于 Stern-Volmer 方程:

$$
I_{0} / I=1+K_{\mathrm{sv}}\left[\mathrm{O}_{2}\right]
$$

其中, $I_{0}$ 和 $I$ 为无氧和含氧状态下的苂光强度, $K_{\mathrm{sv}}$ 为 Stern-Volmer 常数, $\left[\mathrm{O}_{2}\right]$ 为氧分子浓度.

氧分子浓度的增加, 其苂光猝灭程度加大, 指示 剂的荣光强度降低. 图 6 显示, 所构建的氧荣光传感 器对氧的最低检测下限为 $0.2 \mu \mathrm{g} / \mathrm{mL}$, 对氧浓度响应 的线性范围为 $0.5 \sim 16 \mu \mathrm{g} / \mathrm{mL}$, 线性相关系数为 0.9966 . 利用该系统, 对氧浓度 $5.0 \mu \mathrm{g} / \mathrm{mL}$ 的 10 份标准样品进 行连续测定, 所获得的标准偏差为 $2 \%$. 利用该传感 器检测的水体中的溶解氧与商品化仪器(YSI 5100, 美国)所获得的测定数据存在 $2 \%$ 的系统误差.

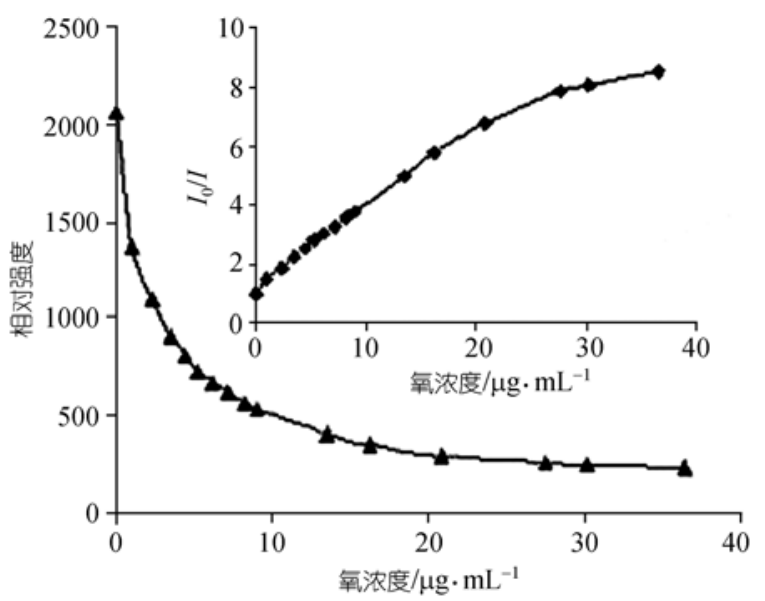

图 6 传感膜对不同溶解氧浓度的苂光响应 插图为图中数据的 Stern-Volmer 方程描绘

\section{3 结论}

利用有机改性溶胶-凝胶方法可以减少传感敏感 膜的极性, 从而增加对敏感膜对氧检测的灵敏度, 减 少响应时间. 实验结果表明, 具有最大激发波长为 $462 \mathrm{~nm}$ 的蓝光 LED, 可以作为溶解氧苂光猝灭测定 的激发光源. 菼光猝灭敏感指示剂在所使用的波长 
下, 其光化学行为稳定. 利用所构建的检测装置, 能 够有效地检测水体中溶解氧的浓度, 氧敏感膜的货 架保存时间不小于 10 个月. 这些结果将对氧光化学 传感膜的制备, 以及氧传感器的研制提供帮助.

致谢本工作为国家高技术研究发展规划(批准号: 2001AA635100)和福建省自然科学基金(批准号：D0410001) 资助项目.

\section{参考文献}

1 Velasco-Garcia N, Valencia-Gonzalez M J, Diaz-Garcia M E. Fluorescent organofilms for oxygen sensing in organic solvents using a fiber optic system. Analyst, 1997, 122(11): 14051409[DOI]

2 Carraway E R, Demas J N, DeGraff B A, et al. Photophysics and photochemistry of oxygen sensors based on luminescent transition-metal complexes. Anal Chem, 1991, 63(4): 337-342[DOI]

3 Rosenzweig Z, Kopelman R. Development of a submicrometer optical fiber oxygen sensor. Anal Chem, 1995(15), 67: 2650[DOI]

4 Klimant I, Wolfbeis O S. Oxygen-sensitive luminescent materials based on silicone-soluble ruthenium diimine complexes. Anal Chem, 1995, 67(18): 3160[DOI]

5 Bossi M L, Daraio M E, Aramendia P F. Luminescence quenching of $\mathrm{Ru}(\mathrm{II})$ complexes in polydimethylsiloxane sensors for oxygen. J Photoch Photobio A, 1999, 120(1): 15-21[DOI]

6 Gillanders R N, Tedford M C, Crilly P J, et al. A composite thin film optical sensor for dissolved oxygen in contaminated aqueous environments. Anal Chim Acta, 2005, 545(2): 189-194[DOI]

7 Tao Z Y, Tehan E C, Tang Y, et al. Stable sensors with tunable sensitivities based on class II xerogels. Anal Chem, 2006, 78(6): 1939[DOI]
8 Chan C M, Chan M Y, Zhang M, et al. The performance of oxygen sensing films with ruthenium- adsorbed fumed silica dispersed in silicone rubber. Analyst, 1999, 124(5): 691—694[DOI]

9 Martin M F C, Dan X. Oxygen-sensitive reverse-phase optode membrane using silica gel-adsorbed ruthenium( II ) complex embedded in gelatin ${ }^{\circledR} \mathrm{lm}$. Anal Chim Acta, 1999, 387(2): 197205[DOI]

10 Chen X, Zhong Z M, Li Z, et al. Characterization of ormosil film for dissolved oxygen-sensing. Sensor Actuat B-Chem, 2002, 87(2): 233-238[DOI]

11 Watkins A N, Wenner B R, Jordan J D, et al. Portable, low-cost, solid-state luminescence-vased $\mathrm{O}_{2}$ sensor. Appl Spectrosc, 1998, 52(2): 750-754[DOI]

12 Klimant I, Ruckruh F, Liebsch G, et al. Fast response oxygen micro-optodes based on novel soluble ormosil glasses. Mikrochim Acta, 1999, 131(1-2): 35-46

13 Murtagh M T, Shahriari M R, Krihak M. A study of the effects of organic modification and processing technique on the luminescence quenching behavior of sol-gel oxygen sensors based on a $\mathrm{Ru}$ (II ) complex. Chem Mater, 1998, 10(12): 3862-3869[DOI]

14 Malins C, Fanni S, Glever H G, et al. The preparation of a sol-gel glass oxygen sensor incorporating a covalently bound fluorescent dye. Anal Commun, 1999, 36(2): 3-4[DOI]

15 蒋亚琪, 钟振明, 陈㬢, 等. 氧对钉 (II) 配合物溶胶-凝胶膜苂 光响应的影响. 光谱学与光谱分析, 2004, 24(7): 844-847

16 McEvoy A K, McDonagh C M, MacCraith B D. Dissolved oxygen sensor based on fluorescence quenching of oxygen-sensitive ruthenium complexes immobilized in sol-gel-derived porous silica coatings. Analyst, 1996, 121(6): 785-788[DOI]

$17 \mathrm{Xu} \mathrm{W}$, McDonough R C, Langsdorf B, et al. Oxygen sensors based on luminescence quenching: Interactions of metal complexes with the polymer supports. Anal Chem, 1994, 66(23): 4133[DOI]

(2006-06-20 收稿, 2006-09-04 接受) 